Résumés des conférences et travaux

\title{
Guy Thuillier (1932-2019)
}

Jean-Michel Leniaud

\section{OpenEdition \\ Journals}

Édition électronique

URL : https://journals.openedition.org/ashp/3326

DOI : $10.4000 /$ ashp.3326

ISSN : 1969-6310

Éditeur

Publications de l'École Pratique des Hautes Études

\section{Édition imprimée}

Date de publication : 1 septembre 2020

Pagination: XVII-XXI

ISSN : 0766-0677

\section{Référence électronique}

Jean-Michel Leniaud, "Guy Thuillier (1932-2019) », Annuaire de l'École pratique des hautes études (EPHE), Section des sciences historiques et philologiques [En ligne], 151 | 2020, mis en ligne le 01 juillet 2020, consulté le 06 juillet 2021. URL : http://journals.openedition.org/ashp/3326 ; DOI : https:// doi.org/10.4000/ashp.3326 


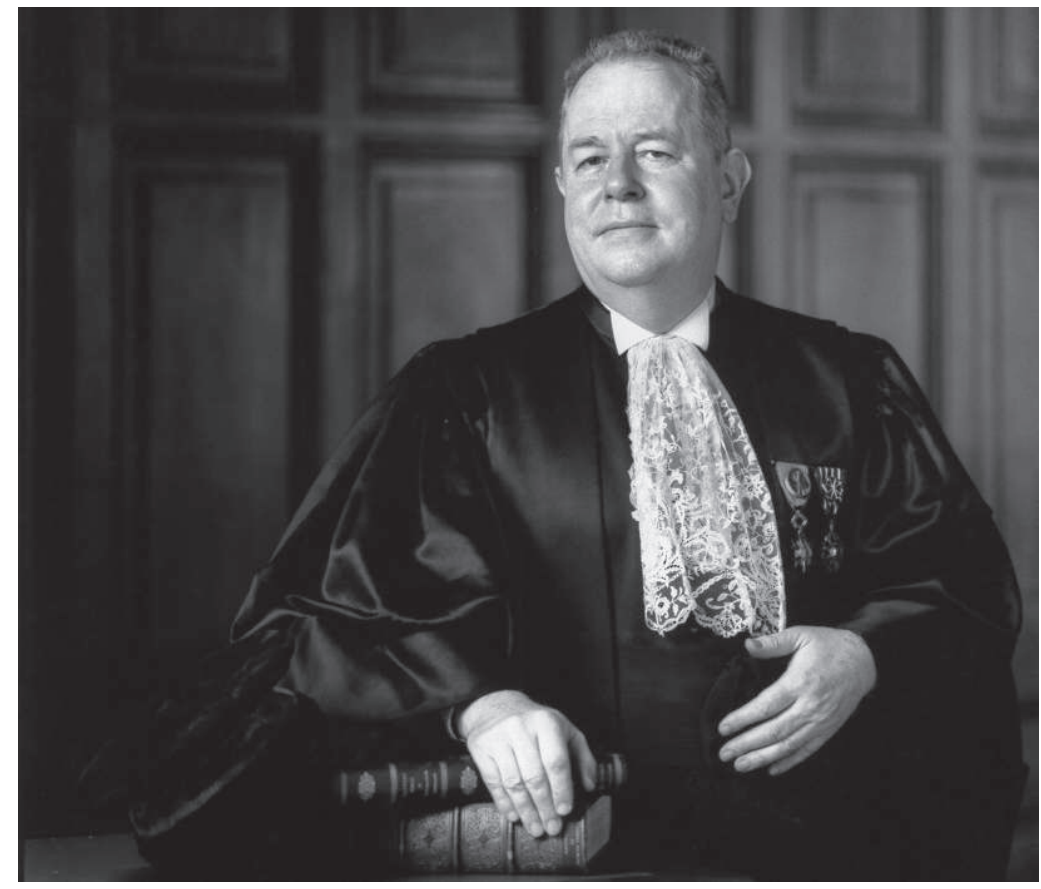

Cliché : Cour des comptes.

\section{GUY THUILLIER}

(1932-2019)

$\mathrm{N}$ otre collègue Guy Thuillier est décédé le 3 mars 2019, à Nevers, ville dans laquelle il s'était retiré depuis la mort de son frère, l'historien de l'art Jacques Thuillier, à l'édition des œuvres duquel il consacrait son temps, son énergie et ses moyens. Diplômé de la section, il avait été chargé de conférence en 1974, selon une tradition qui s'est hélas perdue. Je l'ai connu cette année-là et je suivis son enseignement pendant près de trois ans pour compléter les cours que je recevais alors à l'École des chartes et à Paris IV : sa personnalité me fascina. Né en 1932 à Vaucouleurs, il était entré à l'ÉNA en 1959 puis à la Cour des comptes en 1961 où il acheva sa carrière au grade de conseiller maître. En application d'une disposition de l'époque, le « plan de Constantine », il dut, dès son entrée à la Cour, servir en Algérie, à la préfecture de Saïda d'abord, puis à Alger au gouvernement général. Il m'a souvent parlé avec enthousiasme de cette période. C'est lui qui avait pris l'initiative, sans la moindre couverture hiérarchique, de rapatrier en France les archives de l'administration française, que l'Algérie revendique aujourd'hui; elles se trouvent au centre des Archives de la France d'outre-mer, à Aix-en-Provence. En 1963, il était nommé conseiller technique à la délégation générale à la promotion sociale, puis il servit dans plusieurs cabinets ministériels, comme beaucoup de membres des grands corps dans 
leur jeunesse, de 1968 à 1979, auprès d'Edgar Faure et de Robert Boulin. Pendant cette période, il joua un rôle dans la genèse de la loi sur les handicapés de 1975 et sur les mesures destinées à prévenir la mortalité infantile. Son expérience administrative se nourrissait de ses recherches historiques, sur l'histoire de l'accouchement, par exemple, et réciproquement. J'avais aussi compris de ses propos qu'il avait alors joué un rôle dans l'invention de la procédure de doctorat sur travaux dont bénéficièrent par la suite nombre d'universitaires qui ne venaient pas à bout de leur thèse d'État. Edgar Faure, qu'il suivit jusqu'à la présidence de l'Assemblée nationale, lui confia aussi le soin de documenter sinon de rédiger des parties substantielles de son ouvrage sur la banqueroute de Law. C'est à cette époque que Guy Thuillier commença d'enseigner à la section.

Il était invariablement vêtu de la tenue du haut fonctionnaire de l'époque giscardienne : costume trois pièces de flanelle grise à rayures, cravate sombre, ample pardessus noir. Il enseignait à travailler, à réfléchir, à se documenter, à écrire - il recommandait d'écrire dix pages par jour. Pour ce qui me concerne, je lui dois tout, n'ayant jamais reçu d'aucun de mes maîtres pareil enseignement. Vif, toujours souriant, goguenard, sachant tout sur tout, procédant souvent par allusions, ne citant jamais aucun nom propre dans les conversations, surtout au restaurant, il était doté d'un tel esprit de contradiction qu'il disait déjà « non » avant même qu'on eût esquissé la moindre affirmation. Et si l'on n'était pas convaincu par son dire, il ajoutait d'un air entendu : "Allez voir mon frère! ». Lequel, quand on le rencontrait, disait toujours avec son air de lutin espiègle : «J'espère que vous n'écoutez pas mon frère! ». Toutes ces conversions s'échangeaient au restaurant, deux ou trois cantines où l'un et l'autre invitaient volontiers, mais jamais ensemble. Ils aimaient la table; ils étaient généreux avec leurs étudiants. On pouvait rencontrer notre collègue tous les matins au département des imprimés où il occupait invariablement la même place. Il partait vers quinze heures, "pour la Cour », disait-il. Il se montrait très attaché à la section, plus qu'à l'École, très inquiet des évolutions qui nous ont conduits à ressembler à une université. Il était, par exemple, opposé à la collation du doctorat : « Il faut coller au Collège! », telle était sa formule.

Parmi les innombrables manies de Guy Thuillier, il en était une qu'il faut signaler. Cet homme hyperactif détestait apparaître sous son propre nom : il multipliait les pseudonymes, cosignait des ouvrages qu'il avait parfois écrits presque entièrement de sa plume, en référait invariablement à son frère, s'abritait derrière des personnalités qu'il ne désignait pas. Ainsi, lorsqu'il eut fondé en 1973 le comité pour l'histoire de la sécurité sociale, et qu'il sollicitait des financements, il répétait : " $\mathrm{Si}$ vous ne m'écoutez pas, je vais faire intervenir un vieux monsieur ». Il fallait comprendre qu'il agissait pour le compte du prestigieux Pierre Laroque, fondateur en 1945 de la Sécurité sociale.

Guy Thuillier adorait intervenir dans l'ombre, exercer un pouvoir d'influence à la fois par jeu, par souci d'efficacité, pensait-il : il m'avait fait rencontrer, j'étais élève à l'École des chartes, le conseiller d'État Jacques Narbonne, chargé de rédiger le premier rapport sur ce qu'on appelait alors l'École nationale du patrimoine. Il agissait ainsi par prudence également : son tempérament hypercritique lui avait procuré un nombre substantiel d'ennemis. Il avait ses têtes, en effet : il détestait les archivistes 
auxquels il reprochait de détruire les archives; se moquait des professeurs d'histoire des institutions qui, selon lui, ne comprenaient rien aux modalités du travail administratif; n'aimait pas les chartistes, auxquels il reprochait de cumuler les défauts des deux catégories précédentes, et bien qu'il eût noué des relations très amicales avec plus d'un, Bruno Neveu, Michel Fleury, Jean Valette, Pierre Boisard qui présidait l'UNCAF. Il serait injuste de ne pas signaler ses admirations : Maurice Lévy-Leboyer pour l'histoire de l'économie et Paul Leulliot pour l'histoire de la vie quotidienne.

Et, il faut le noter, il aimait jouer un rôle qui amusait ceux qui en avaient l'habitude, en affectant un mélange de rondeur et de suffisance. La bibliothèque de la Cour des comptes conserve de lui un portrait photographique, revêtu du sévère habit noir du magistrat avec le rabat de dentelle que son frère lui avait offert : tout dans la pose fait penser à un fermier général portraituré par Rigaud ou Largillière, mais pourtant ravi, goguenard, espiègle. Sans compter qu'en ces temps d'après Soixantehuit qui classaient le monde universitaire selon les règles du western, les gauchistes et les « fachos », il avait choisi son camps. Permettez-moi de relater ce petit dialogue caractéristique : lorsqu'en 1976, je lui annonçait mon succès au concours de l'inspection des monuments historiques et qu'après avoir longuement théorisé sur les différences entre les fonctions d'exécution et de contrôle, il me demanda : «Et vous, que comptez-vous faire? Quel type d'inspecteur serez-vous? Vous comptez vous faire aimer? ». Je lui répondis que je souhaitais faire aimer les monuments historiques. Et lui me dit : «Aimer? Mais vous êtes gauchiste! ».

La production de Guy Thuillier est immense : une quarantaine d'ouvrages et des centaines d'articles. Sans compter les publications sous pseudonyme et les entreprises concrètes : en 1973, la création du comité d'histoire de la sécurité sociale; en 1987, le comité d'histoire économique et financière du ministère des Finances. L'un et l'autre servirent de modèle à de nombreux comités ultérieurs, au Quai d'Orsay et ailleurs. Ajoutons l'animation de la Revue administrative et aussi les colloques annuels qu'au nom de notre section, avec le président Michel Fleury, à partir de 1975, il co-organisait chaque année dans la salle d'assemblée générale du Conseil d'État avec l'Institut français des sciences administratives. C'est depuis la section qu'en effet, il produisit l'essentiel de son œuvre. Il y avait été élu directeur d'études en 1980 après six ans de charge de conférence, il y retrouvait un autre spécialiste d'histoire financière, Michel Bruguière (1939-1989). Quatre axes pourraient orienter le lecteur dans un ensemble de recherches foisonnant si on laisse de côté quelques publications plus techniques, sur la drogue (avec Yves Pélicier) ou sur la politique du travail. Le premier rassemble des réflexions épistémologiques sur l'histoire, non seulement sur les méthodes de recherche et de travail mais sur la durabilité des résultats. Il était obsédé par la sénescence des recherches et par le devoir d'innovation. Le petit garçon de dix ans qui, pendant l'Occupation, décrochait des murs de Nevers les placards affichés par l'occupant pour les offrir ultérieurement aux Archives départementales est resté toute sa vie marqué par les conditions de production de l'histoire de demain : d'où la création de comités d'histoire dans les institutions publiques et ses entreprises d'enquêtes orales. En 2002, il publia L'Histoire et le probabilisme. Mais il ne s'agissait pas seulement de sources à inventer : pour lui, l'historien cherchait par son travail, si peu que ce fût, à vaincre la mort, celle des sociétés, en tentant de faire revivre l'objet 
même de son étude, mais peut-être aussi la sienne propre, par la durabilité de ses travaux. Thuillier alimentait son exercice d'introspection sur l'art d'écrire l'histoire par une lecture approfondie des auteurs spirituels du XVII ${ }^{\mathrm{e}}$ siècle.

Le deuxième axe concerne le travail concret de l'historien. Ce qu'il a écrit dans plusieurs petits livres de la collection «Que sais-je? » en compagnie de Jean Tulard reste d'une utilité quotidienne pour les jeunes chercheurs : le choix d'un sujet et la manière de l'aborder - il insistait sur la nécessité de choisir un sujet « technique ", plus difficile et moins fréquenté; le choix des sources, la manière d'en prendre connaissance, d'y noter les informations, de les rassembler, etc.; l'art d'écrire, de constituer des index ${ }^{1}$ - il privilégiait les index matière - , de rédiger un compte rendu d'ouvrage. Bref, il faisait entrer le novice dans son propre atelier et le formait à son art, échangeait des savoir-faire avec ses jeunes pairs et multipliait les conseils. Il adorait donner des conseils.

Le troisième axe concerne son projet d'histoire de l'administration. Il récusait les analyses d'organigramme et détestait les approches trop juridiques : il s'était fait l'anthropologue de l'administration. Le travail au quotidien, l'organisation d'un bureau, les ambitions, les rêveries, les maladies de l'agent administratif, l'arrivée des femmes aux différents niveaux de la fonction publique, la figure du conseiller technique, jeune loup dont la carrière s'accélère par un passage dans un cabinet ministériel, les corps de fonctionnaires, voilà qui l'intéressait. Il procédait à la manière néo-impressionniste, par petites touches juxtaposées. Il en résulta de nombreux ouvrages, sur la haute administration (1979), sur les cabinets ministériels (1982), sur l'imaginaire quotidien (1985), sur la vie quotidienne dans les ministères au XIX ${ }^{\mathrm{e}}$ siècle (2004) pour n'en citer que quelques-uns.

Le quatrième axe concerne l'histoire locale. Il privilégiait volontairement et vigoureusement l'approche monographique qui lui permettait la pluridisciplinarité. Bien que Lorrain de naissance, Guy Thuillier avait fait de la Nièvre sa patrie d'adoption et son sujet d'études. Dans la suite de son maître Paul Leulliot, il enquêta sur le quotidien nivernais au XIX ${ }^{\mathrm{e}}$ siècle, en multipliant les approches les plus innovantes, non plus seulement l'histoire du capitalisme sidérurgique auquel il avait consacré son premier ouvrage ou des domestiques (1978), mais celle de l'eau, de l'accouchement, du vêtement, de la lessive, du bruit, de l'arrivée du stylo à bille et ajoutons encore, celle de Bernadette Soubirous au sujet de laquelle il rédigea un guide de recherches archivistiques en 2008. L'histoire du quotidien, soulignait-il, multipliait les défis en termes de sources et d'écriture historienne. Et surtout, elle forçait, disait-il encore, à faire le grand écart entre l'histoire de l'État central et parisien et celle du local. Pas d'historien digne de ce nom, pensait-il, sans histoire locale. Thuillier fut le soutien constant d'une association nivernaise, la Camosine, tournée vers l'histoire et les patrimoines locaux. L'œuvre historienne de Guy Thuillier est énorme. Certains ont cru y voir du disparate, la marque d'un esprit papillonnant. C'était ne rien comprendre à son projet : nourrir l'histoire par une démarche anthropologique et l'analyse simultanée des deux poumons de la société française, l'État central et la vie locale. Il procédait par petites touches, à la fois par goût et en raison du fait qu'il vivait

1. C'est lui qui avait établi l'index de la thèse de doctorat d'État de Maurice Lévy-Leboyer. 
une seconde vie professionnelle, celle des cabinets ministériels puis de la Cour des comptes : la première se développa dans les interstices laissés par la seconde. Elle fut distinguée par quelques récompenses académiques : quatre prix de l'Institut, la médaille d'argent du CNRS et le titre de membre correspondant de l'académie des sciences morales et politiques. Il ne voulait pas s'y présenter car son frère, disait-il, refusait de le faire à l'Académie des inscriptions et belles-lettres. Pour Guy Thuillier, pas d'œuvre historienne sans expérience professionnelle. Il s'intéressait à (presque) tout : histoire sociale, histoire du travail, histoire locale, histoire des femmes, histoire du quotidien, histoire de la monnaie, histoire de la métallurgie, histoire des députés, histoire de l'administration... Il était fécond, et rendait tels ceux qui l'approchaient. Ses disciples sont nombreux.

Jean-Michel LenIAUd 